\title{
Gilteritinib: A Novel FLT3 Inhibitor for Relapsed/Refractory Acute Myeloid Leukemia
}

\author{
DENNIS MARJONCU, ${ }^{1}$ PharmD, BCOP, and BENJAMIN ANDRICK, ${ }^{2}$ PharmD, BCOP
}

From Methodist University Hospital, Memphis, Tennessee; '2Geisinger Medical Center, Danville, Pennsylvania

Authors' disclosures of conflicts of interest are found at the end of this article.

Correspondence to: Dennis Marjoncu, PharmD, 1265 Union Avenue, Memphis, TN 38104. E-mail: dennis.marjoncu@mlh.org https://doi.org/10.6004/jadpro.2020.11.1.7 (c) 2020 Harborside $^{T M}$

\begin{abstract}
Acute myeloid leukemia ( $A M L$ ) is the most common adult leukemia, with an overall poor prognosis. New agents targeting various receptors may improve treatment outcomes and overall survival. FMS-like tyrosine kinase 3 (FLT3) is a targetable mutation occurring in one third of AML patients. It contributes to increased tumor proliferation and decreased cellular differentiation, ultimately conferring a poor overall prognosis. Among patients with FLT3-positive relapsed/refractory AML, outcomes are particularly dismal. Gilteritinib is a novel, secondgeneration FLT3 inhibitor approved by the U.S. Food \& Drug Administration (FDA) for the treatment of relapsed/refractory AML with an FLT3 mutation as detected by an FDA-approved test.
\end{abstract}

cute myeloid leukemia (AML) is the most common adult leukemia, with an estimated incidence of 21,450 patients in 2019. Additionally, mortality remains high, with an estimated 10,920 deaths occurring in 2019 (American Cancer Society, 2019). The underlying pathophysiology is driven by clonal proliferation of immature myeloid precursor cells secondary to chromosomal translocations and molecular mutations (De Kouchkovsky \& Abdul-Hay, 2016). Recent advances have identified several key mutations that play a role in AML.

One targetable mutation in the treatment of AML involves the FMS- like tyrosine kinase 3 (FLT3) protein. Coded on chromosome 13q12 and normally expressed on hematopoietic stem cells, the FLT3 protein promotes cellular communication for the differentiation of myeloid precursor cells to their mature counterparts (Daver, Schlenk, Russell, \& Levis, 2019). Mutations of this signaling protein typically manifest in one of two ways. The FLT3 internal tandem duplication (ITD) subtype occurs in $30 \%$ of AML patients. It is associated with an increased risk of relapse and decreased overall survival (OS) compared to those without the mutation (Sutamtewagul \& Vigil, 2018). Studies have further stratified the poor 
prognosis of this mutation based on the allelic ratio, between mutant and wild-type, as greater than 0.5 (Sutamtewagul \& Vigil, 2018). Alternatively, the FLT3 tyrosine kinase domain (TKD) mutation, most often a missense mutation, is a constitutive change of the tyrosine kinase domain. The prognostic significance is not yet known, but ongoing studies seek to identify its significance (Grafone, Palmisano, Nicci, \& Storti, 2012).

The historical standard treatment for AML involved remission induction with cytarabine plus an anthracycline, followed by consolidation therapy involving high-dose cytarabine and potentially an allogeneic hematopoietic stem cell transplant (National Comprehensive Cancer Network, 2019). Following the elucidation of FLT3 mutation's involvement in the pathogenesis of AML, targeted inhibitors were developed to enhance the armamentarium of treatment options beyond conventional chemotherapy. Midostaurin (Rydapt), the first FLT3 inhibitor to receive U.S. Food \& Drug Administration (FDA) approval, is recommended in combination with an anthracycline and cytarabinebased induction. It is dosed $50 \mathrm{mg}$ orally twice daily on days 8 to 21 and subsequently with each cycle of cytarabine-based consolidation (National Comprehensive Cancer Network, 2019; Sutamtewagul \& Vigil, 2018). In the RATIFY trial, midostaurin plus chemotherapy improved OS compared to placebo (median 74.7 vs. 25.6 months; $p=.009$; Stone et al., 2017). The RATIFY trial also included midostaurin maintenance for up to 1 year; however, this dosing is not FDA approved due to the lack of efficacy seen in the trial (Sutamtewagul \& Vigil, 2018).

Given the success of midostaurin to alter disease trajectory, several FLT3 inhibitor compounds are being investigated as therapeutic alternatives for the treatment of AML. Gilteritinib (Xospata), the most recently FDA-approved FLT3 inhibitor, is the first second-generation FLT3 inhibitor compound available on the market. Gilteritinib is indicated for use in adults with relapsed/refractory AML with an FLT3 mutation detected by an FDAapproved test (Astellas Pharma Inc, 2018).

\section{MECHANISM OF ACTION}

Mutated FLT3 receptors are activated via dimerization and phosphorylation independently of ligands, resulting in unrestricted downstream signaling and cell proliferation (Grafone et al., 2012). Gilteritinib is a second-generation tyrosine kinase inhibitor that inhibits several kinases, including FLT3, AXL, ALK, and c-kit (Lee et al., 2017; Figure 1). Specific to FLT3 inhibition, gilteritinib uniquely targets both the ITD and TKD mutations, including FLT3-ITD, FLT3-ITD-D835Y, and FLT3-D835Y. Gilteritinib ultimately induces apoptosis in cells expressing FLT3-ITD (Astellas Pharma Inc, 2018).

\section{CLINICAL TRIALS}

Perl and colleagues (2017) conducted a phase I/ II study to evaluate the safety, tolerability, and pharmacokinetics of gilteritinib in adults with relapsed/refractory AML. The trial included 252 adults with primary relapsed/refractory or secondary AML and an Eastern Cooperative Oncology Group (ECOG) performance status (PS) of $\leq$ 2. While the presence of the FLT3 mutation was not an explicit inclusion criterion, at least 10 patients in each dose level were required to have a confirmed FLT3 mutation (ITD or TKD). Patients who received three or more lines of prior therapy (44\%) and had FLT3-ITD mutation status (64\%) composed the majority of the study population (Perl et al., 2017). Dose escalation was conducted in seven cohorts utilizing a $3 \times 3$ design of oncedaily dosing: $20 \mathrm{mg}, 40 \mathrm{mg}, 80 \mathrm{mg}, 120 \mathrm{mg}, 200$ $\mathrm{mg}, 300 \mathrm{mg}$, and $450 \mathrm{mg}$. The maximum tolerated

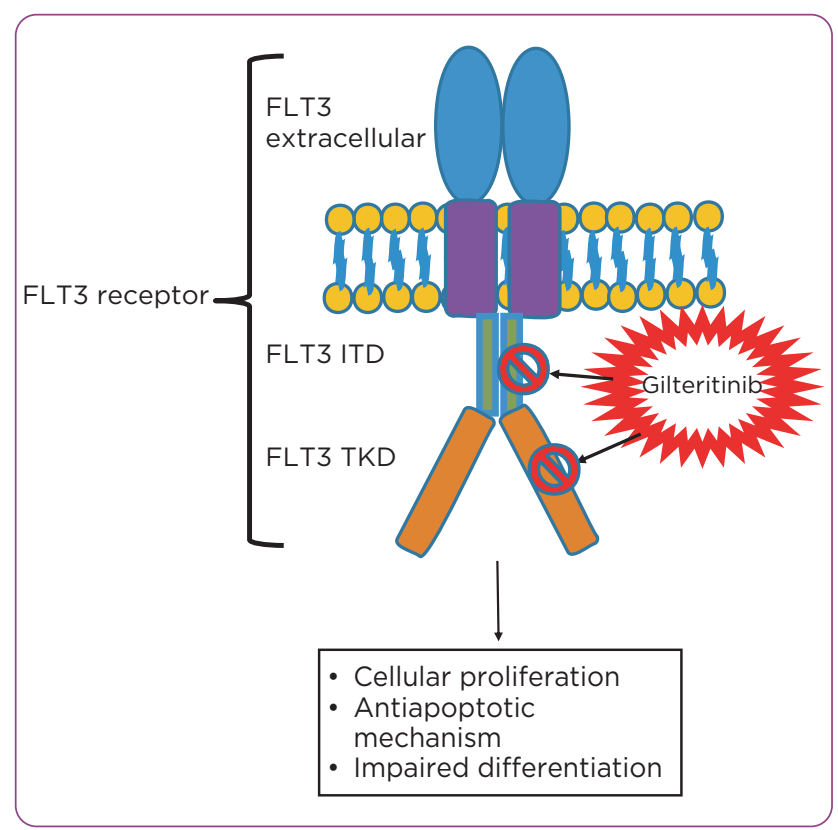

Figure 1. Gilteritinib mechanism of action. 
dose in the phase I portion was $300 \mathrm{mg}$ daily, with two out of three patients experiencing a dose-limiting toxicity with $450 \mathrm{mg}$ daily dosing (one case of grade 3 diarrhea and one case of grade 3 elevated aspartate aminotransferase).

In vivo inhibition of FLT3 phosphorylation correlated with increasing doses, as demonstrated by patients receiving $80 \mathrm{mg}$ with at least $90 \%$ FLT3 inhibition (Perl et al., 2017). Antileukemic effect was evident in all dose cohorts. Of these, 19 (8\%) achieved a complete response (CR), 10 (4\%) achieved CR with incomplete platelet recovery, 46 (18\%) achieved CR with incomplete hematologic recovery (CRh), and 25 (10\%) achieved a partial response. Ultimately, 100 (40\%) of this heavily pretreated relapsed/refractory population achieved a response. Of the 75 patients achieving CR, 36 (48\%) patients received $120 \mathrm{mg}$ daily. Such results supported the selection of $120 \mathrm{mg}$ as the target dose utilized in the phase III study (Perl et al., 2017).

Perl and colleagues subsequently conducted the phase III ADMIRAL trial to evaluate gilteritinib vs. salvage chemotherapy in 371 adults with relapsed/refractory FLT3-positive AML. The salvage chemotherapy comparator arm was selected at the providers' discretion between high-intensity chemotherapy with MEC (mitoxantrone, etoposide, and cytarabine), FLAG-IDA (fludarabine, cytarabine, idarubicin, and granulocyte colony-stimulating factor), or low-intensity chemotherapy of low-dose cytarabine or azacytidine. Both treatment arms continued therapy until disease progression.

The CR/CRh rate was $34 \%$ with gilteritinib vs. $15.3 \%$ in those receiving investigator's choice of salvage chemotherapy. The median time to response was 1.8 months in those receiving gilteritinib and 1.1 months receiving salvage chemotherapy. Furthermore, the median duration of response was 11 months with gilteritinib compared to 1.8 months with salvage therapy. Of those patients who achieved a CR/CRh in the gilteritinib arm, all had FLT3-ITD \pm TKD. Interestingly, no patients with FLT3-TKD mutation alone achieved a CR/ CRh. Ultimately, the median OS was 9.3 months in those assigned to the gilteritinib arm compared to 5.6 months in those receiving salvage chemotherapy. Additionally, the 1-year survival rate was $37.1 \%$ in the gilteritinib arm and $16.7 \%$ in the chemotherapy arm. 106 patients in the gilteritinib arm were dependent on red blood cell and/or platelet transfusions at baseline. Of these, 33 (31.1\%) became transfusion independent after 56 days. An additional 32 patients in the gilteritinib arm were independent of transfusions at baseline. Of these, 17 (53.1\%) patients remained transfusion independent during the follow-up period (Perl et al., 2019).

\section{ADVERSE EFFECTS}

The most common treatment-related adverse effects seen in the ADMIRAL trial were anemia (33\%), elevated aspartate aminotransferase (24\%) and alanine aminotransferase (24\%), febrile neutropenia (21\%), and thrombocytopenia (19\%; Perl et al., 2019). A full list of adverse effects occurring in more than $10 \%$ of patients from the gilteritinib studies can be found in Table 1 .

Additionally, the prescribing information for gilteritinib contains warnings for posterior reversible encephalopathy syndrome (PRES), prolonged QTc intervals, pancreatitis, and embryo-fetal toxicity (Astellas Pharma Inc, 2018).

\section{DOSING AND ADMINISTRATION}

Gilteritinib is approved for adults with relapsed/ refractory AML with an FLT3 mutation as detected by an FDA-approved test. The starting dose is $120 \mathrm{mg}$ (three $40 \mathrm{mg}$ tablets) orally, with or without food, administered at the same time once daily until disease progression or unacceptable toxicity. It is recommended to treat for a minimum of 6 months to allow time for a clinical response. Blood counts, serum chemistries, and creatine phosphokinase should be monitored prior to the initiation of therapy. Follow-up should occur at least weekly for the first month, every other week for the second month, and once monthly thereafter. Electrocardiogram (ECG) should be obtained prior to initiation, on days 8 and 15 of the first cycle, and prior to the start of cycles two and three. Women of reproductive potential should have a negative pregnancy documented within the first 7 days of treatment initiation. A summary of monitoring parameters is provided in Table 2 . Patients should be monitored for signs and symptoms of pancreatitis and PRES (Astellas Pharma Inc, 2018).

The package insert provides dose adjustments for "other grade 3 or higher toxicities," such as anemia and thrombocytopenia. The recommen- 


\begin{tabular}{|lll|}
\hline \multicolumn{2}{|c|}{$\begin{array}{c}\text { Table 1. Select Treatment-Related Adverse Events } \\
\text { of Gilteritinib }\end{array}$} \\
\hline Adverse event & Any grade & Grade 3-4 \\
Arthralgia/myalgia & $42 \%$ & $5 \%$ \\
Dizziness & $20 \%$ & $<1 \%$ \\
Dyspnea & $34 \%$ & $12 \%$ \\
Edema & $34 \%$ & $2 \%$ \\
Fatigue & $40 \%$ & $5 \%$ \\
Noninfectious diarrhea & $34 \%$ & $3 \%$ \\
Pneumonia & $30 \%$ & $23 \%$ \\
Rash & $30 \%$ & $3 \%$ \\
Transaminitis & $41 \%$ & $16 \%$ \\
\hline
\end{tabular}

dations are to hold gilteritinib until the toxicity improves to grade 1 , then resume treatment at a lower dose of $80 \mathrm{mg}$ (2 tablets; Astellas Pharma Inc, 2018).

Patients should not crush or chew the tablet. Missed doses should be administered as soon as possible on the same day and at least 12 hours prior to the next dose. Patients should not take two doses within 12 hours (Astellas Pharma Inc, 2018).

\section{IMPLICATIONS FOR THE ADVANCED PRACTITIONER}

Gilteritinib is the second FLT3 inhibitor approved for the treatment of AML. Unlike midostaurin, gilteritinib is currently approved for FLT3-positive disease in the relapsed/refractory setting. In this setting, gilteritinib offers a survival benefit that is approximately two-fold greater than salvage chemotherapy. Additionally, gilteritinib adds to patient quality of life since it is administered orally to facilitate outpatient treatment. It is important to note that gilteritinib only benefits patients with FLT3-positive AML, therefore supporting the practice that all patients be tested for FLT3 positivity prior to initiating therapy. Practitioners should be aware of the frequent lab and ECG monitoring parameters for patients initiating gilteritinib therapy, particularly in the first 3 months.

At the starting dose of $120 \mathrm{mg}$ daily, patients will take three tablets per dose, as it is only available in the 40-mg tablet strength. There are no specific dose adjustments for renal (creatinine clearance $<30 \mathrm{~mL} /$ minute) or hepatic dysfunction (Child-Pugh class C); however, it has not been

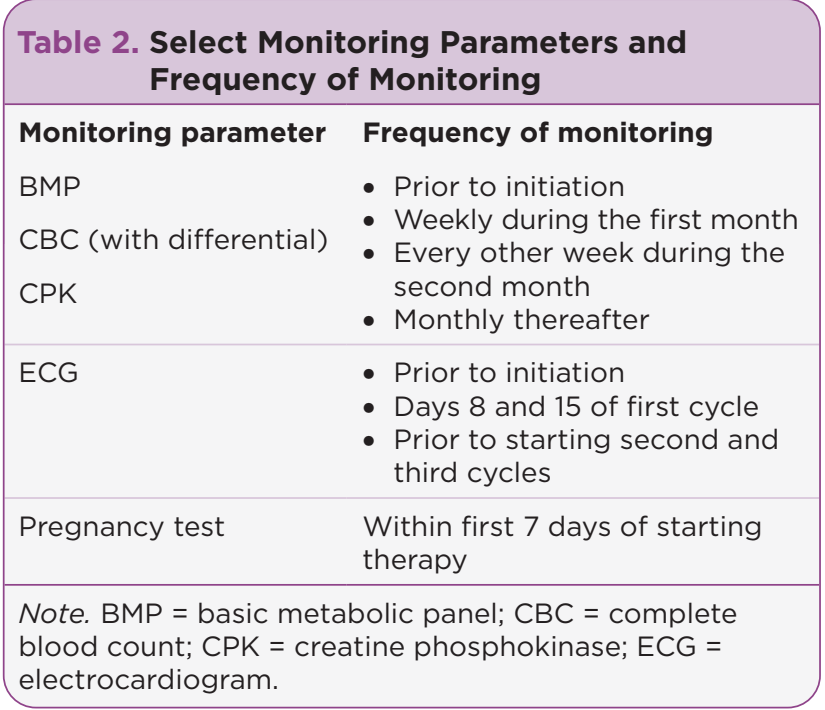

studied in these populations. Caution should be exercised when determining possible treatment for these patients (Astellas Pharma Inc, 2018).

Rare cases of pancreatitis and PRES have been reported. If a patient experiences pancreatitis, treatment should be held until the pancreatitis resolves, and gilteritinib should be resumed at a dose of $80 \mathrm{mg}$ once daily. Patients who experience PRES while on gilteritinib should permanently discontinue therapy. Reports of QTc interval prolongation have been reported. If the QTc interval increases beyond greater than $500 \mathrm{~ms}$, gilteritinib should be held. Therapy should be resumed at a reduced dose of $80 \mathrm{mg}$ daily once the QTc interval returns to $\leq 480 \mathrm{~ms}$ or is within $30 \mathrm{~ms}$ of baseline. During cycle 1, if the QTc interval increases by $>30 \mathrm{~ms}$ on the day $8 \mathrm{ECG}$, a repeat ECG should be conducted on day 9. If the QTc interval remains prolonged, gilteritinib should immediately be reduced to $80 \mathrm{mg}$ daily (Astellas Pharma Inc, 2018).

The most common adverse effects seen in clinical trials were cytopenias and hepatic enzyme elevation. Like midostaurin, dermatologic rash can occur with gilteritinib, likely due to off-target effects. Gilteritinib is metabolized via the CYP3A4 pathway; therefore, patients should be screened for potentially significant drug interactions with other medications. If the use of strong CYP3A4 inhibitors cannot be avoided, patients should be monitored more closely for side effects associated with gilteritinib therapy. Additionally, patients should avoid the use of combined P-glycoprotein 
(P-gp) and strong CYP3A4 inducers with concurrent gilteritinib use.

Based on the success of gilteritinib in the relapsed/refractory setting, several studies are underway to evaluate its role in other phases of care. One trial is evaluating gilteritinib in combination with azacytidine in newly diagnosed FLT3positive AML in adults who cannot tolerate standard chemotherapy (ClinicalTrials.gov, 2019a). There is also a trial evaluating midostaurin vs. gilteritinib in previously untreated patients with newly diagnosed AML in combination with standard induction and consolidation (ClinicalTrials.gov, 2019b).

A recent poster presented by Zeidan and coleagues (2019) at the ASH Annual Meeting determined that, compared to salvage chemotherapy, gilteritinib led to an increase of 1.55 discounted life-years and an increase of 1.29 discounted quality-adjusted life-years (QALY). This results to an additional cost of $\$ 141,097$ relative to salvage chemotherapy, translating to an incremental cost per life-year gained of $\$ 90,761$, and an incremental cost per QALY of $\$ 109,741$. Thus, gilteritinib is a costeffective strategy based on the $\$ 150,000$ per QALY threshold recommended by the Institute for Clinical and Economic Review (Zeidan et al., 2019).

\section{CONCLUSION}

Patients with relapsed/refractory AML have historically had limited treatment options. Often treatment required the use of high-dose salvage chemotherapy. Gilteritinib is a targeted therapy that provides an alternative for this high-risk population and has demonstrated improved efficacy. The adverse effect profile largely consists of hepatic enzyme elevations and cytopenias, although these can be effectively managed. Gilteritinib offers a novel treatment with manageable adverse effects for patients with FLT3-positive AML who have relapsed/refractory disease.

\section{Disclosure}

The authors have no conflicts of interest to disclose.

\section{References}

American Cancer Society. (2019). Key statistics for acute myeloid leukemia. Retrieved from https://www.cancer.org/ cancer/acute-myeloid-leukemia/about/key-statistics.html Astellas Pharma Inc. (2018). Xospata (gilteritinib) tablets package insert. Retrieved from https://www.accessdata. fda.gov/drugsatfda_docs/label/2018/211349s000lbl.pdf

ClinicalTrials.gov. (2019a). A Study of ASP2215 (Gilteritinib) by Itself, ASP2215 Combined With Azacitidine or Azacitidine by Itself to Treat Adult Patients Who Have Recently Been Diagnosed With Acute Myeloid Leukemia With a FLT3 Gene Mutation and Who Cannot Receive Standard Chemotherapy.

ClinicalTrials.gov. (2019b). A Study of Gilteritinib Versus Midostaurin in Combination With Induction and Consolidation Therapy Followed by One-year Maintenance in Patients With Newly Diagnosed Acute Myeloid Leukemia or Myelodysplastic Syndromes With Excess Blasts-2 With FLT3 Mutations Eligible for Intensive Chemotherapy (HOVON 156 AML).

Daver, N., Schlenk, R. F., Russell, N. H., \& Levis, M. J. (2019). Targeting FLT3 mutations in AML: Review of current knowledge and evidence. Leukemia, 33(2), 299-312. https://doi.org/10.1038/s41375-018-0357-9

De Kouchkovsky, I., \& Abdul-Hay, M. (2016). Acute myeloid leukemia: A comprehensive review and 2016 update. Blood Cancer Journal, 6(7), e441. https://doi.org/10.1038/ bcj.2016.50

Grafone, T., Palmisano, M., Nicci, C., \& Storti, S. (2012). An overview on the role of FLT3-tyrosine kinase receptor in acute myeloid leukemia: Biology and treatment. Oncology Reviews, 6(1), e8. https://doi.org/10.4081/oncol.2012.e8

Lee, L. Y., Hernandez, D., Rajkhowa, T., Smith, S. C., Raman, J. R., Nguyen, B.,...Levis, M. (2017). Preclinical studies of gilteritinib, a next-generation FLT3 inhibitor. Blood, 129(2), 257-260. https://doi.org/10.1182/blood-2016-10-745133

National Comprehensive Cancer Network. (2019). NCCN Clinical Practice Guidelines in Oncology: Acute Myeloid Leukemia. V2.2019.

Perl, A. E., Altman, J. K., Cortes, J., Smith, C., Litzow, M., Baer, M. R.,...Levis, M. (2017). Selective inhibition of FLT3 by gilteritinib in relapsed or refractory acute myeloid leukaemia: A multicentre, first-in-human, open-label, phase 1-2 study. Lancet Oncology, 18(8), 1061-1075. https://doi. org/10.1016/S1470-2045(17)30416-3

Perl, A. E., Martinelli, G., Cortes, J. E., Neubauer, A., Berman, E., Paolini, S.,...Levis, M. J. (2019). Abstract CT184 Gilteritinib significantly prolongs overall survival in patients with FLT3-mutated relapsed/refractory acute myeloid leukemia: Results from the phase III ADMIRAL trial. Cancer Research, 79(13 suppl). https://doi. org/10.1158/1538-7445.AM2019-CT184

Stone, R. M., Mandrekar, S. J., Sanford, B. L., Laumann, K., Geyer, S., Bloomfield, C. D.,...Döhner, H. (2017). Midostaurin plus chemotherapy for acute myeloid leukemia with a FLT3 mutation. New England Journal of Medicine, 377(5), 454-464. https://doi.org/10.1056/NEJMoal614359

Sutamtewagul, G., \& Vigil, C. E. (2018). Clinical use of FLT3 inhibitors in acute myeloid leukemia. OncoTargets and Therapy, 11, 7041-7052. https://doi.org/10.2147/OTT. S171640

Zeidan, A. M., Qi, C. Z., Pandya, B. J., Garnham, A., Yang, H., \& Shah, M. V. (2019). Cost-effectiveness analysis of gilteritinib versus salvage chemotherapy (SC) for the treatment of relapsed or refractory FLT3-mutated acute myeloid leukemia. Poster session presented at the Annual Convention of the American Society of Hematology. 